Meta

Journal des traducteurs

Translators' Journal

\title{
Réflexions sur un examen
}

\section{Irène V. Spilka}

Volume 21, numéro 3, septembre 1976

URI : https://id.erudit.org/iderudit/004256ar

DOI : https://doi.org/10.7202/004256ar

Aller au sommaire du numéro

Éditeur(s)

Les Presses de l'Université de Montréal

ISSN

0026-0452 (imprimé)

1492-1421 (numérique)

Découvrir la revue

Citer cet article

Spilka, I. V. (1976). Réflexions sur un examen. Meta, 21(3), 188-196.

https://doi.org/10.7202/004256ar

Ce document est protégé par la loi sur le droit d'auteur. L'utilisation des services d'Érudit (y compris la reproduction) est assujettie à sa politique d'utilisation que vous pouvez consulter en ligne.

https://apropos.erudit.org/fr/usagers/politique-dutilisation/
Cet article est diffusé et préservé par Érudit.

Érudit est un consortium interuniversitaire sans but lucratif composé de l'Université de Montréal, l'Université Laval et l'Université du Québec à Montréal. Il a pour mission la promotion et la valorisation de la recherche. https://www.erudit.org/fr/ 


\section{Réflexions sur un examen}

Passer un examen n'est déjà pas chose facile, mais le corriger...! Entre cent versions d'un même passage, laquelle choisir ? et d'après quels critères ? Existe-t-il seulement des critères sûrs? Des souvenirs d'école nous reviennent en mémoire: écrire en «bon français», "ça ne doit pas sentir la traduction», il faut être «fidèle » (oui, mais à qui, à quoi ?). Pour en finir, et par souci de méthode, nous avons résolu d'appliquer rigoureusement le premier principe que nous proposons chaque année à la réflexion des étudiants, comprendre avant de traduire. Pour cela, nous disposons d'un certain nombre d'instruments. Les dictionnaires sont là pour nous révéler le sens des mots obscurs ou franchement inconnus. Mais un texte n'est pas la somme des mots qui le composent. Il faut saisir la trame des relations qui unissent les mots entre eux, et cela, c'est l'affaire de la syntaxe. Enfin, on doit pouvoir relier les mots et leur agencement syntaxique aux choses; autrement dit, faire correspondre le texte et la réalité. Cette dernière opération qui, de fait, est la première dans l'ordre de la compréhension spontanée, exige du lecteur qu'il connaisse le sujet traité, aucune analyse lexicale ou syntaxique ne pouvant, en effet, suppléer à l'ignorance de la matière.

Si les candidats qui désirent faire reconnaître leur aptitude par une société de traducteurs sont des gens cultivés, souvent doublés de spécialistes, et s'ils savent se servir d'un dictionnaire sans commettre les bévues qui font la joie des ramasseurs de perles, il leur arrive parfois, tout comme aux étudiants en traduction, de rester perplexes devant les détours et les méandres de la phrase anglaise, dont la syntaxe et la stylistique diffèrent de celles du français. C'est pourquoi nous avons pensé leur rendre service en faisant avec eux une analyse un peu poussée des textes proposés lors d'un examen.

Cette analyse s'inspire, disons-le sans détour, de la grammaire générative transformationnelle. Mais, qu'on se rassure, le bref exposé qui va suivre ne renferme ni termes abscons ni raisonnements hermétiques. Simplement, on cherche à mettre en évidence les rapports des divers constituants de la phrase en vue d'aider le traducteur à franchir la première étape de son travail, celle de l'interprétation du texte, qui précède obligatoirement la recherche des équivalences et la rédaction définitive de la version française. Pour ne pas lasser le lecteur, et parce que quelques phrases suffisent à illustrer la méthode, nous nous contentons d'étudier le 
début du premier texte proposé à l'examen, le plus général des trois, donc le plus susceptible d'intéresser l'ensemble des candidats reçus, refusés et éventuels. Voici le passage étudié :

For the professional translator, one of the most exasperating aspects of translating modern texts on a variety of subjects is the inordinate amount of searching and learning time he has to expend to evaluate special terms employed by the authors of source language documents.

On peut aborder cette phrase complexe par le début, c'est-à-dire en la lisant de la façon normale, de gauche à droite. Une telle lecture livre un premier segment qui, heureusement pour nous, se trouve délimité par une majuscule et une virgule (For the professional translator,). Ce segment ne constitue pas cependant un début de phrase normal, mais la suite logique de quelque autre segment. C'est précisément la recherche de ce segment-là qui a fait achopper certains candidats, car, s'ils saisissaient bien le sens global du passage, ils ne parvenaient pas à trouver le membre de phrase auquel il fallait rattacher d'une façon très précise, afin de faire une phrase convenablement articulée en français, la préposition for et sa suite (ce que les linguistes appellent parfois un «syntagme prépositionnel »). Plutôt que de les inviter à relire normalement de gauche à droite, nous proposont aux candidats une opération différente : projeter la phrase sur un schéma préexistant, le schéma d'une phrase type. Ce schéma représente une pure abstraction, exactement comme le chiffre 2 est la représentation d'une abstraction, le nombre deux, par rapport à une couple d'œufs ou à une paire de bas. Il correspond vraisemblablement à un schème mental, celui d'un ensemble de règles syntaxiques servant à organiser les sons et les signes que nous percevons (les mots) pour nous permettre de leur donner un sens. Sous sa forme élémentaire, le schéma, appelé «arbre », se présente ainsi :

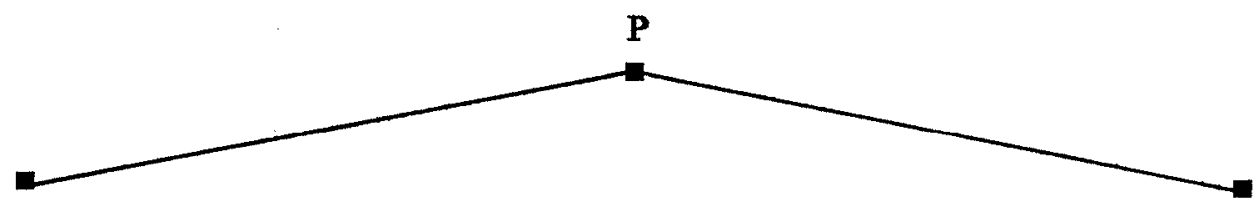

Le sommet $\mathbf{P}$ correspond à la phrase ou proposition entière, en fait, on ne les distingue pas, car elles ont la même structure) ; chacune des branches aboutit à un nœud qui constitue l'un des éléments essentiels de la phrase. Celui de gauche (le syntagme nominal) désigne la place du sujet, et celui de droite (le syntagme verbal) celle du verbe et de sa suite éventuelle. Certaines phrases comptent trois branches à partir du sommet; dans ce cas, la troisième, qui peut précéder ou suivre les deux autres, désigne le lieu d'un complément circonstanciel de phrase.

Projetons la phrase $\mathbf{P}$ sur le schéma arborescent. Elle se divise naturellement en deux parties :

1. For the professional translator, one of the most exasperating aspects of translating modern texts on a variety of subjects 
2. is the inordinate amount of searching and learning time he has to expend to evaluate special terms employed by the authors of source language documents.

La première partie est le syntagme nominal (SN) de la phrase, ou si l'on préfère une terminologie plus traditionnelle, le sujet. La seconde partie en est le syntagme verbal (SV), c'est-à-dire le verbe ou la copule et sa suite. Chacune de ces parties se laisse à son tour décomposer en un certain nombre d'éléments plus petits. Les décompositions successives du schéma se font à partir des derniers nœuds obtenus, dont on dit qu'ils dominent les branches suivantes. Le nœud appelé SN domine le plus souvent les rameaux du nom (N) et du déterminant (DET), qui peut être un article, un adjectif démonstratif, ou un adjectif numéral, tous facultatifs en anglais. Il peut également dominer des phrases, complément de nom ou bien proposition relative, qui sont dites enchâssées. L'arbre prend alors l'un des aspects suivants :

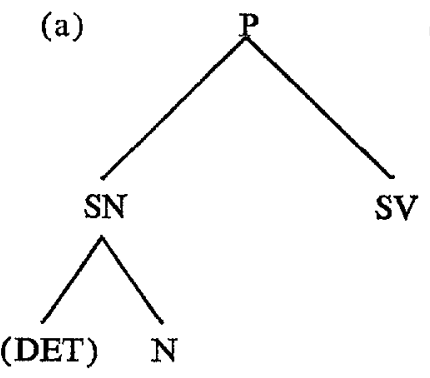

(b)

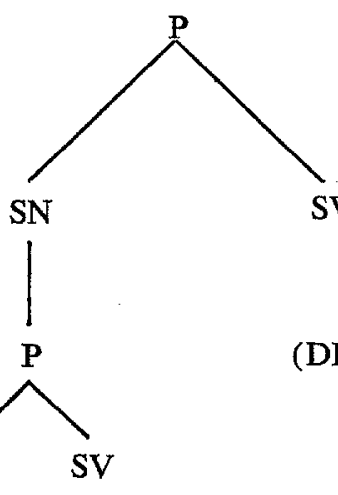

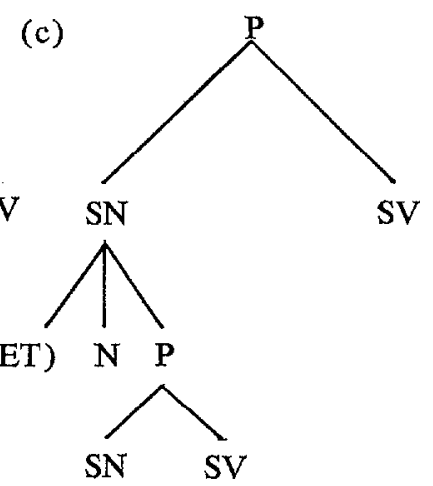

Cherchons parmi les mots du premier membre de phrase celui qui peut jouer le rôle de sujet. Il est clair que ce mot ne peut être que one, puisque c'est le seul élément singulier, à part translator qui appartient, nous l'avons déjà dit, à une suite et non à un début de phrase. Par ailleurs, one, qui joue ici le rôle d'un pronom (cf. One Flew over the Cuckoo's Nest), est doté d'un long complément : of the most exasperating aspects of translating modern texts on a variety of subjects, qui appelle à son tour une analyse. Comme la tournure one of the most... ne présente guère de difficulté (il s'agit, on l'aura compris, d'une forme abrégée de *one of the aspects that are the most exasperating aspects... ${ }^{1}$, nous ne nous y attarderons pas. Par contre, il est intéressant d'étudier l'entourage de aspects : 1) exasperating, 2) of translating modern texts on a variety of subjects.

Curieusement, ces deux constituants comportent chacun une forme en -ing; or, la forme anglaise en -ing risque d'être ambiguë car elle peut jouer deux rôles, celui d'épithète et celui de gérondif. Exasperating est sans doute employé ici comme

1. L'astérisque indique un passage sous-jacent, présumé, mais non réalisé; il peut également indiquer que le passage est fautif. 
épithète puisqu'on peut dire these aspects are (most) exasperating sans changer le sens de la phrase. En fait, les grammairiens modernes croient que l'épithète provient d'une relative enchâssée, ce que l'on admet aisément si l'on pense à des cas d'effacement comme le garçon est malade $\rightarrow$ le malade. Mais les adjectifs semblent parfois se comporter comme des verbes, puisqu'ils peuvent être suivis du complément d'agent, par exemple, introduit par une préposition: le lion malade de la peste (C'est la peste qui rend le lion malade). Cette propriété de l'adjectif éclaire singulièrement notre phrase, et la fonction syntaxique de for the professional translator devient évidente. The most exasperating aspects... provient d'une phrase sousjacente (c'est-à̀-dire un modèle de phrase présumé mais non réalisé) qui serait la suivante: *these aspects are the most exasperating for the professional translator. L'épithète qualifiant translator provient, comme exasperating, d'une phrase attributive sous-jacente, * the translator is professional.

Le second complément de aspects, of translating modern texts on a variety of subjects, est d'une autre nature. Translating esț sûrement un gérondif, puisqu'il est suivi d'un objet direct, modern texts. Or, le gérondif provient du verbe, et à ce titre suppose la présence d'un sujet - qui peut évidemment être exprimé ou demeurer tacite. Il est permis de supposer que l'action décrite par translating est exécutée par un agent qui soit le translator. Il était sans doute inutile de le répéter dans la phrase anglaise, puisque ce concept apparaissait déjà dans le syntagme complément d'exasperating, mais il est important de le garder en mémoire, car, au moment de la traduction, il pourrait être souhaitable, pour des raisons d'ordre stylistique, de construire la phrase autour d'un nom d'agent humain plutôt qu'à partir d'un nom de chose.

On vient de voir que l'entourage d'un nom, aspects, se composait de phrases sous-jacentes que, pour la commodité, on abrège considérablement, par un procédé d'effacement, dans la réalisation finale de la phrase parlée ou écrite, réalisation dite $\ll$ structure superficielle $》$.

On peut démontrer de façon analogue que texts donne lieu à deux phrases sous-jacentes: 1) these texts are modern, 2) these texts are on a variety of subjects. Ces procédés, effacement et enchâssement, sont propres au langage humain ; au reste, ils n'apparaissent que graduellement au cours de l'enfance. Chez l'adulte, surtout chez l'écrivain habile, ils permettent d'arriver à une grande concision dans l'expression. Mais, au moment de traduire, le traducteur ne doit pas se laisser leurrer par l'aspect définitif, extérieur, superficiel, de la phrase, car celui-ci recouvre des faits complexes qu'il importe de mettre en lumière pour pénétrer le texte avant de le restituer sous une forme différente.

Le deuxième membre de phrase; le SV, peut se diviser en deux et comporter un verbe $\mathrm{V}$ (copule, auxiliaire, verbe transitif, voire un adjectif fonctionnant comme un verbe) et un SN, qui, tout comme le SN situé à gauche du verbe suit toutes les règles gouvernant les syntagmes nominaux dans une langue donnée. On peut donc rencontrer à droite, dans le schéma, des développements comme ceux-ci : 


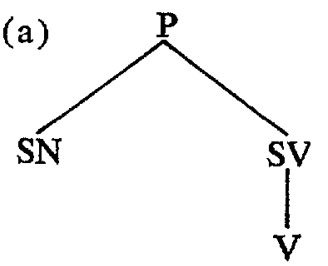

(b)

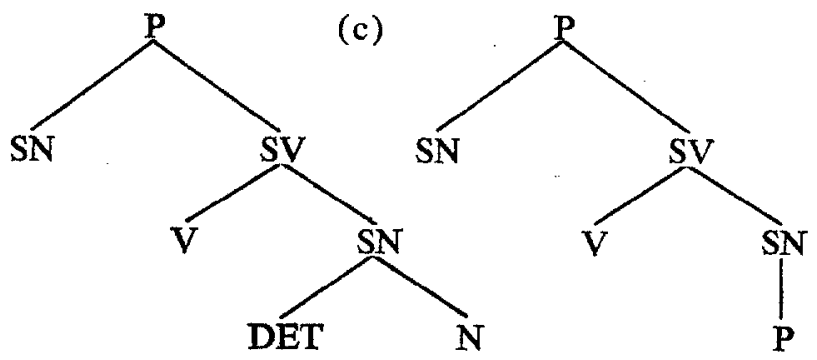

Dans le cas présent, nous projetons is sur V et la suite sur SN (dans le schéma b). Procédant comme tout à l'heure, nous trouvons dans l'entourage du nom amount les phrases sous-jacentes *this amount is inordinate, *this amount is of time. Par contre, searching time et learning time sont des expressions plus difficiles à analyser selon le schéma classique. En effet, on sait que logiquement, time n'est pas le sujet de searching, mais le complément d'une phrase qui serait à peu près ceci : *someone spends time searching ou encore: *someone searches during a time. Une telle hypothèse est certes trop vague pour satisfaire les théoriciens, mais elle peut rendre service au traducteur, en le forçant à examiner la nature du lien qui unit l'épithète en -ing au nom qu'elle qualifie.

Par ailleurs, time est suivi d'une relative enchâssée : he has to expend... sans pronom relatif apparent, ce qui est possible en anglais. Cette relative peut correspondre au schéma arborescent qui suit :

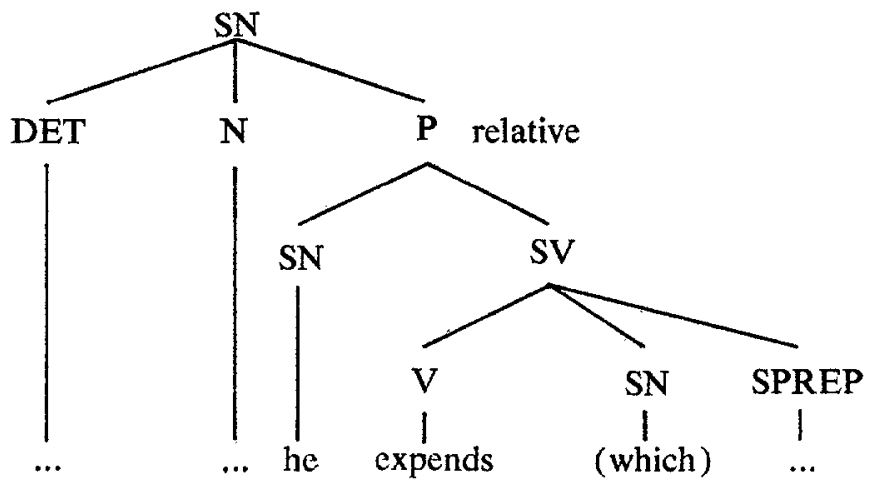

Sous le nœud du syntagme prépositionnel on peut faire figurer une subordonnée enchâssée, en l'occurrence to evaluate special terms, qui exprime la fin vers laquelle tend l'action. Son sujet n'étant pas défini, on peut le remplacer par *someone, mais nous savons par ailleurs qu'il s'agit toujours du même agent (c'est la condition nécessaire de son effacement), le translator du début de la phrase complexe. Quant à terms, qui est qualifié par special (*these terms are special), et employed (*these terms are employed by the authors...), il va nous fournir l'occasion d'analyser une phrase passive.

La phrase passive, en anglais comme en français, est le résultat d'une transformation qui ajoute un auxiliaire et une préposition, et qui transporte l'agent à gauche et l'objet à droite, selon le très classique exemple de la souris mangée par 
le chat. En rétablissant la forme active, dans le cas qui nous intéresse, nous obtenons * the authors... employ these terms, qui est une relative enchâssée sous le SN complément d'objet de to evaluate :

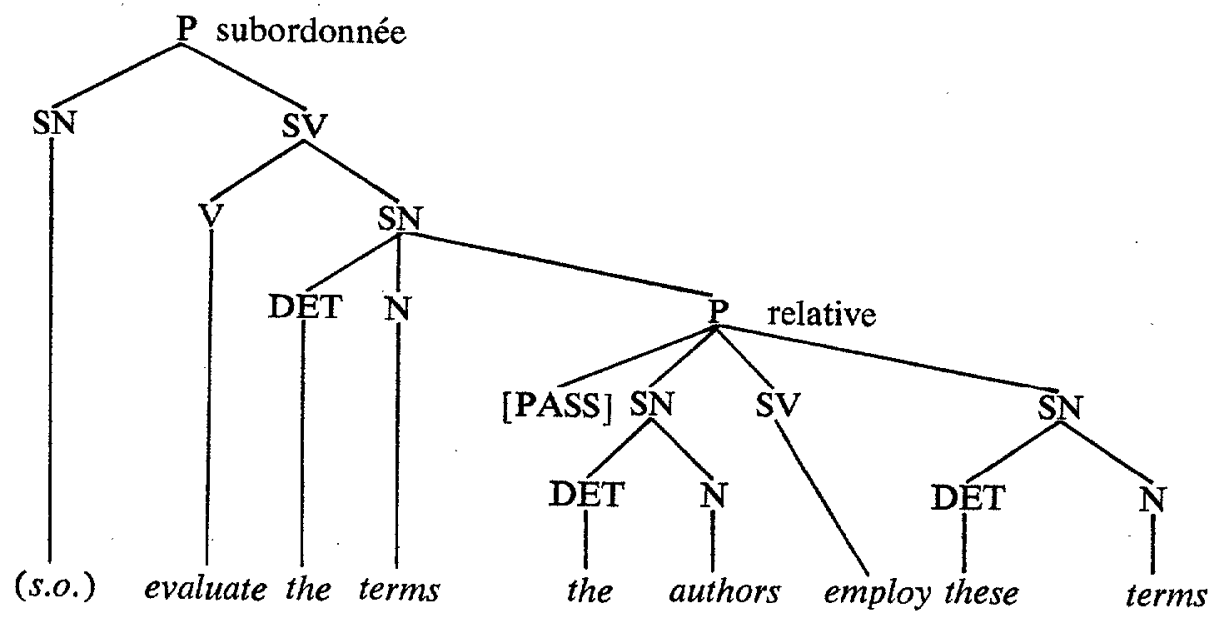

La phrase se termine par une cascade de compléments, que l'anglais a la propriété de rejeter à gauche dans la structure superficielle, ... of source language documents. On comprend que le dernier nom, documents, est le complément de authors : the authors of... documents; il est à son tour modifié par source language : *documents are written in the source language, ce qui, en rétablissant la forme active donne *the authors write the documents in the source language. Enfin, language est luimême modifié par source : *this language is the source.

Récapitulons. Nous venons de faire une lecture analytique d'un passage. Plutôt que de respecter l'ordre dans lequel les mots se présentent sur le papier, nous avons cherché ça et là dans le passage les éléments correspondant aux repères fournis par les schémas de la grammaire générative transformationnelle et nous avons tenté de leur assigner la place qui leur revient dans la hiérarchie des constituants qui s'unissent pour former une phrase anglaise. Les spécialistes nous pardonneront si nous avons erré ou pris de trop grandes libertés dans l'interprétation de la théorie. Notre seul objectif est d'amener le traducteur débutant, hésitant, ou déconcerté par une phrase difficile, à rompre avec les vieilles formules dans l'espoir de trouver parmi les nouvelles un instrument de travail mieux adapté aux besoins de la traduction. Voici ce que livre notre découpage : une série de phrases courtes reconstituées à partir des données explicites ou tacites du texte original qui serviront à refaire un texte, français cette fois.

1. * One of the aspects is the amount of time

2. *These aspects are most exasperating for the translator

3. *These aspects are (phrase 4)

4. * Someone (the translator) translates texts

5. *These texts are modern

6. * These texts are on a variety of subjects 


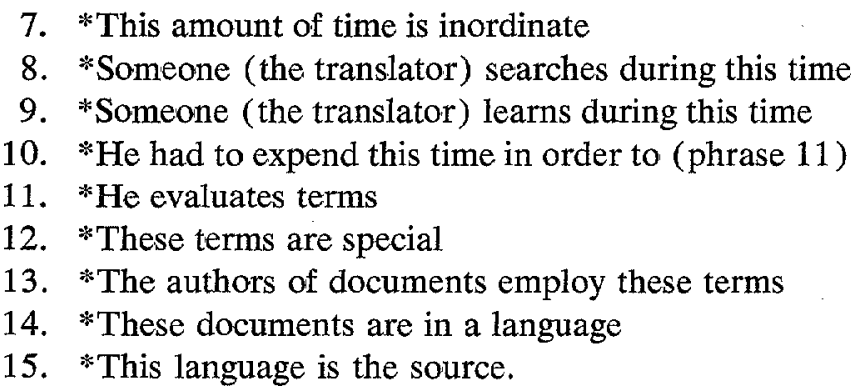

L'établissement de cette série clôt la première étape du travail, celle de la compréhension raisonnée du texte. Lorsque les circonstances l'exigent, on doit pousser plus loin le raisonnement et «interpréter »le texte; il y aurait beaucoup à dire sur l'interprétation des textes à traduire, mais c'est une question à laquelle nous reviendrons une autre fois. La deuxième étape peut maintenant commencer, la recherche des équivalences. Le moment est venu de fouiller dans sa mémoire, ou dans le dictionnaire, qui est la mémoire de la langue, pour retrouver les mots français qui servent à exprimer les idées contenues dans le texte. Ces idées sont rarement d'une originalité telle qu'il n'existe pas de mot pour les dire. En fait la plupart des idées, concepts, notions, que véhiculent le français et l'anglais ont quelque chose d'universel - à tout le moins, d'occidental - qui fait qu'ils ont depuis longtemps trouvé leur formule linguistique. Les nouveautés surgissent surtout dans le domaine concret des objets. Le peuple ne s'embarrasse guère de soucis linguistiques pour nommer ce qui lui tombe sous les sens; il emprunte ( $d u$ coke), décrit (un porte-crayons avec un calendrier mobile incorporé), évoque (bleu faïence), compare (une chenille, en parlant d'un véhicule), bref il invente les mots dont il a besoin. Les terminologues viennent ensuite sanctionner ses créations; mais ils tardent parfois et les traducteurs ne savent s'ils doivent imiter le peuple ou attendre les décisions des spécialistes. Ce qui constitue une problème d'ordre normatif. Quant aux choses, et aux idées (car il y en a) qui sont entièrement nouvelles, leur expression est un défi lancé à l'intelligence et à l'initiative de celui qui écrit, fût-il auteur ou simple traducteur. Dans le cas que nous étudions, rien de tel. Les mots clés (que l'on peut toujours souligner en rouge, afin de ne pas en oublier) sont fort courants : aspect, amount of time, exasperating, translator, translate (-ing), texts, modern, variety, subjects, inordinate, search (-ing), learn (-ing), expend, evaluate, term, special, author, document, employ, language, source. Il n'y a pas là de quoi émouvoir même un néophyte. Quant aux relations entre les termes, elles ont été révélées par l'analyse. Les phrases qui en résultent pourraient être traduites une à une de manière à former un canevas (assez semblable à ceux que l'on nous proposait jadis dans les cours de rédaction) à partir duquel s'élaborerait le travail de composition qui livrera le texte définitif. La composition d'un tel canevas est utile lorsqu'on se trouve aux prises avec un texte difficile. Elle ne s'impose pas dans le cas présent, nous passerons donc sans transition à l'établissement d'un premier texte, un avant-texte, qui offre déjà des phrases plus longues et plus complexes que ne le ferait la première traduction, celle des phrases marquées d'un astérisque, mais qui ne se présente pas encore sous une forme prête à livrer au lecteur. Voici donc ce premier jet : 
- Certains aspects de la traduction des textes modernes exaspèrent le traducteur professionnel.

- Les textes modernes portent sur des sujets très divers.

- L'un de ces aspects, c'est le temps consacré par le traducteur à l'évaluation de certains termes.

- Les auteurs des documents originaux emploient, en effet, des termes spécialisés.

- Le traducteur consacre un temps fort long à la recherche et à l'étude de ces termes.

Tout y est - on peut s'en assurer en comparant l'avant-texte à la liste des mots clés anglais - mais cela n'est pas dit d'une façon très élégante. Il reste une dernière étape à franchir, celle de la rédaction du texte, et c'est là que se manifestent l'habileté, le talent et les goûts personnels du traducteur. C'est l'étape où l'art vient embellir les réussites de la science et de la technique.

Il existe évidemment bien des manières de re-dire (nous décomposons le mot à dessein) le message de l'auteur, et toutes se valent dans les limites étroites de la fidélité.

Car tout est là : restituer le message qui nous a été confié, à nous traducteurs, par l'auteur, par son représentant, ou par le lecteur, qui nous fait confiance et qui nous demande de lui dire ce que l'auteur a dit. Nous sommes pendant un temps dépositaires d'un bien qui ne nous appartient pas ; la propriété en revient au seul auteur, mais c'est le lecteur qui en a la jouissance. D'où la triple origine de la fidélité en traduction, fidélité au message, à son auteur, et à son lecteur éventuel. D'où aussi l'obligation de comprendre avant d'écrire, et d'effectuer un travail de re-composition conscient et raisonné.

Parmi les versions soumises à notre examen, plusieurs se recommandaient à l'attention du correcteur par leur excellente qualité. Nous voudrions les présenter toutes, mais cela n'est guère possible dans le cadre d'un article de revue. Par ailleurs, nous nous en voudrions de n'en choisir qu'une, laissant croire aux autres candidats que la leur était moins intéressante, aussi avons-nous cru pouvoir contourner la difficulté en composant un portrait robot à l'aide des meilleures copies. C'est le produit de cet effort collectif que nous ajoutons en annexe, en remerciant les sociétés et les candidats qui nous ont donné l'occasion de réfléchir en corrigeant l'examen.

IRÈNE V. SPILKA

\section{ANNEXE I}

For the professional translator, one of the most exasperating aspects of translating modern texts on a variety of subjects is the inordinate amount of searching and learning time he has to expend to evaluate special terms employed by the authors of source language documents. It has been claimed that up to $60 \%$ of a conscientious translator's work time is consumed in tracking down proper terminological information. 
Not only is the volume of new terminology increasing rapidly; but also the looseness of using the technical vocabulary is growing among various specialists in the field. Added to the proliferation of unabbreviated specialized terminology must be the ever-enlarging usage of acronyms and initialisms which frequently cannot be decoded even by experts in a given field without the aid of a proper dictionary. However, even the most up-to-date printed dictionary cannot maintain a rate of speed parallel with the burgeoning growth of specialized terminology.

\section{ANNEXE II}

L'un des aspects les plus exaspérants de la traduction de textes modernes portant sur toutes sortes de sujets, c'est le temps excessif que le traducteur professionnel est obligé de consacrer à la recherche et à l'étude afin d'interpréter convenablement les termes spécialisés employés par les auteurs des documents originaux. On a même prétendu qu'un traducteur consciencieux consacrait jusqu'à $60 \%$ de son temps à la recherche terminologique.

Non seulement le nombre des néologismes augmente-t-il rapidement, mais l'imprécision du vocabulaire technique se répand chez les spécialistes de divers domaines. À la prolifération des termes techniques s'ajoute l'emploi de plus en plus fréquent des acronymes et des sigles, que les spécialistes eux-mêmes ne savent pas toujours déchiffrer sans le secours d'un dictionnaire spécialisé. Or, le dictionnaire imprimé même le plus souvent remis à jour se laisse dépasser par la croissance rapide de la terminologie technique. 